\title{
On Dilation Operators in Besov Spaces
}

\author{
Cornelia SCHNEIDER \\ Leipzig University \\ Department of Mathematics \\ PF 100920 \\ D-04009 Leipzig - Germany \\ schneider@math.uni-leipzig.de
}

Received: April 22, 2008

Accepted: May 6, 2008

\begin{abstract}
We consider dilation operators $T_{k}: f \rightarrow f\left(2^{k} \cdot\right)$ in the framework of Besov spaces $B_{p, q}^{s}\left(\mathbb{R}^{n}\right)$ when $0<p \leq 1$. If $s>n\left(\frac{1}{p}-1\right), T_{k}$ is a bounded linear operator from $B_{p, q}^{s}\left(\mathbb{R}^{n}\right)$ into itself and there are optimal bounds for its norm. We study the situation on the line $s=n\left(\frac{1}{p}-1\right)$, an open problem mentioned in $[5,2.3 .1,2.3 .2]$. It turns out that the results shed new light upon the diversity of different approaches to Besov spaces on this line, associated to definitions by differences, Fourier-analytical methods, and subatomic decompositions.
\end{abstract}

Key words: Besov spaces, dilation operators, moment conditions.

2000 Mathematics Subject Classification: $46 \mathrm{E} 35$.

\section{Introduction}

In this article we consider dilation operators of the form

$$
T_{k} f(x)=f\left(2^{k} x\right), \quad x \in \mathbb{R}^{n}, \quad k \in \mathbb{N},
$$

which represent bounded operators from $B_{p, q}^{s}\left(\mathbb{R}^{n}\right)$ into itself. Their behaviour is well known when $s>\sigma_{p}=n \max \left(\frac{1}{p}-1,0\right)$. In this situation we have, for $0<p, q \leq \infty$,

$$
\left\|T_{k} \mid \mathcal{L}\left(B_{p, q}^{s}\left(\mathbb{R}^{n}\right)\right)\right\| \sim 2^{k\left(s-\frac{n}{p}\right)}, \quad s>\sigma_{p},
$$

The author was supported by DFG Graduiertenkolleg (Research Training Group) 597 
see [5]. We study the dependence of the norm of $T_{k}$ on $k$ on the line $s=\sigma_{p}$, where $0<p \leq 1$. In particular, we obtain for $0<q \leq \infty$ that

$$
\left\|T_{k} \mid \mathcal{L}\left(B_{p, q}^{\sigma_{p}}\left(\mathbb{R}^{n}\right)\right)\right\| \sim 2^{k\left(\sigma_{p}-\frac{n}{p}\right)} k^{1 / q} .
$$

The situation when $s=0$ was already investigated in [18, sec. 3], where it was proved that, for $0<q \leq \infty$,

$$
\left\|T_{k} \mid \mathcal{L}\left(B_{p, q}^{0}\left(\mathbb{R}^{n}\right)\right)\right\| \sim 2^{-k \frac{n}{p}} \cdot \begin{cases}k^{\frac{1}{q}-\frac{1}{\max (p, q, 2)}}, & \text { if } 1<p<\infty, \\ k^{\frac{1}{q}}, & \text { if } p=1 \text { or } p=\infty .\end{cases}
$$

We generalize the methods (and adapt the notation) used there.

As a by-product the new results for the dilation operators lead to new insights concerning the nature of the different approaches to Besov spaces - namely the classical $\left(\mathbf{B}_{p, q}^{s}\right)$, the Fourier-analytical $\left(B_{p, q}^{s}\right)$ and the subatomic approach $\left(\mathfrak{B}_{p, q}^{s}\right)-$ on the line $s=\sigma_{p}$.

So far recent results by Hedberg, Netrusov [7] on atomic decompositions and by Triebel [17, sec. 9.2] on the reproducing formula prove coincidences

$$
\mathbf{B}_{p, q}^{s}\left(\mathbb{R}^{n}\right)=\mathfrak{B}_{p, q}^{s}\left(\mathbb{R}^{n}\right), \quad s>0, \quad 0<p, q \leq \infty,
$$

as subspaces of $L_{p}\left(\mathbb{R}^{n}\right)$ and

$$
B_{p, q}^{s}\left(\mathbb{R}^{n}\right)=\mathbf{B}_{p, q}^{s}\left(\mathbb{R}^{n}\right)=\mathfrak{B}_{p, q}^{s}\left(\mathbb{R}^{n}\right), \quad s>\sigma_{p}, \quad 0<p, q \leq \infty,
$$

(in terms of equivalent quasi-norms) in $S^{\prime}\left(\mathbb{R}^{n}\right)$. Furthermore, since for $s<n\left(\frac{1}{p}-1\right)$ the $\delta$-distribution belongs to $B_{p, q}^{s}\left(\mathbb{R}^{n}\right)$ - which is a singular distribution and cannot be interpreted as a function - the spaces

$$
B_{p, q}^{s}\left(\mathbb{R}^{n}\right) \quad \text { and } \quad \mathbf{B}_{p, q}^{s}\left(\mathbb{R}^{n}\right), \quad 0<s<\sigma_{p},
$$

cannot be compared. The situation on the line $s=\sigma_{p}, 0<p<1$, so far remained an open problem. If $q>1$ there are singular distributions belonging to $B_{p, q}^{\sigma_{p}}\left(\mathbb{R}^{n}\right)$. Hence $B_{p, q}^{\sigma_{p}}\left(\mathbb{R}^{n}\right)$ and $\mathbf{B}_{p, q}^{\sigma_{p}}\left(\mathbb{R}^{n}\right)$ cannot be compared. If $q \leq 1$ then $\mathbf{B}_{p, q}^{\sigma_{p}}\left(\mathbb{R}^{n}\right)$ is a subspace of $L_{1}^{\text {loc }}\left(\mathbb{R}^{n}\right)$ and the two spaces $B_{p, q}^{\sigma_{p}}\left(\mathbb{R}^{n}\right)$ and $\mathbf{B}_{p, q}^{\sigma_{p}}\left(\mathbb{R}^{n}\right)$ can be compared. But our results yield that they do not coincide, i.e.,

$$
B_{p, q}^{\sigma_{p}}\left(\mathbb{R}^{n}\right) \neq \mathbf{B}_{p, q}^{\sigma_{p}}\left(\mathbb{R}^{n}\right), \quad 0<p<1, \quad 0<q \leq 1,
$$

as sets of measurable functions, where $\mathbf{B}_{p, q}^{\sigma_{p}}\left(\mathbb{R}^{n}\right)$ can be replaced by $\mathfrak{B}_{p, q}^{\sigma_{p}}\left(\mathbb{R}^{n}\right)$ in view of (2). 


\section{Besov spaces $B_{p, q}^{s}\left(\mathbb{R}^{n}\right)$}

We use standard notation. Let $\mathbb{N}$ be the collection of all natural numbers and let $\mathbb{N}_{0}=\mathbb{N} \cup\{0\}$. Let $\mathbb{R}^{n}$ be an Euclidean $n$-space, $n \in \mathbb{N}, \mathbb{C}$ the complex plane. The set of multi-indices $\beta=\left(\beta_{1}, \ldots, \beta_{n}\right), \beta_{i} \in \mathbb{N}_{0}, i=1, \ldots, n$, is denoted by $\mathbb{N}_{0}^{n}$, with $|\beta|=\beta_{1}+\cdots+\beta_{n}$, as usual. Moreover, if $x=\left(x_{1}, \ldots, x_{n}\right) \in \mathbb{R}^{n}$ and $\beta=\left(\beta_{1}, \ldots, \beta_{n}\right) \in \mathbb{N}_{0}^{n}$ we put $x^{\beta}=x_{1}^{\beta_{1}} \cdots x_{n}^{\beta_{n}}$.

We use the equivalence ' $\sim$ ' in

$$
a_{k} \sim b_{k} \quad \text { or } \quad \varphi(x) \sim \psi(x)
$$

always to mean that there are two positive numbers $c_{1}$ and $c_{2}$ such that

$$
c_{1} a_{k} \leq b_{k} \leq c_{2} a_{k} \quad \text { or } \quad c_{1} \varphi(x) \leq \psi(x) \leq c_{2} \varphi(x)
$$

for all admitted values of the discrete variable $k$ or the continuous variable $x$, where $\left\{a_{k}\right\}_{k},\left\{b_{k}\right\}_{k}$ are non-negative sequences and $\varphi, \psi$ are non-negative functions. If $a \in \mathbb{R}$, then $a_{+}:=\max (a, 0)$ and $[a]$ denotes the integer part of $a$.

All unimportant positive constants will be denoted by $c$, occasionally with subscripts. For convenience, let both $\mathrm{d} x$ and $|\cdot|$ stand for the (n-dimensional) Lebesgue measure in the sequel. As we shall always deal with function spaces on $\mathbb{R}^{n}$, we may usually omit the ' $\mathbb{R}^{n}$ ' from their notation for convenience.

Let $Q_{\nu, m}$ with $\nu \in \mathbb{N}_{0}$ and $m \in \mathbb{Z}^{n}$ denote a cube in $\mathbb{R}^{n}$ with sides parallel to the axes of coordinates, centred at $2^{-\nu} m$, and with side length $2^{-\nu+1}$. For a cube $Q$ in $\mathbb{R}^{n}$ and $r>0$, we denote by $r Q$ the cube in $\mathbb{R}^{n}$ concentric with $Q$ and with side length $r$ times the side length of $Q$.

Furthermore, when $0<p \leq \infty$, the number $\sigma_{p}$ is given by

$$
\sigma_{p}=n\left(\frac{1}{p}-1\right)_{+}
$$

\section{The Fourier-ANALYTiCAL APPROACH}

The Schwartz space $\mathcal{S}\left(\mathbb{R}^{n}\right)$ and its dual $\mathcal{S}^{\prime}\left(\mathbb{R}^{n}\right)$ of all complex-valued tempered distributions have their usual meaning here. Let $\varphi_{0}=\varphi \in \mathcal{S}\left(\mathbb{R}^{n}\right)$ be such that

$$
\operatorname{supp} \varphi \subset\left\{y \in \mathbb{R}^{n}:|y|<2\right\} \quad \text { and } \quad \varphi(x)=1 \quad \text { if }|x| \leq 1 \text {, }
$$

and for each $j \in \mathbb{N}$ let $\varphi_{j}(x)=\varphi\left(2^{-j} x\right)-\varphi\left(2^{-j+1} x\right)$. Then $\left\{\varphi_{j}\right\}_{j=0}^{\infty}$ forms a smooth dyadic resolution of unity. Given any $f \in \mathcal{S}^{\prime}\left(\mathbb{R}^{n}\right)$, we denote by $\hat{f}$ and $f^{\sim}$ its Fourier transform and its inverse Fourier transform, respectively. If $f \in \mathcal{S}^{\prime}\left(\mathbb{R}^{n}\right)$, then the compact support of $\varphi_{j} \hat{f}$ implies by the Paley-Wiener-Schwartz theorem that $\left(\varphi_{j} \hat{f}\right)^{\sim}$ is an entire analytic function on $\mathbb{R}^{n}$. 
Definition 1.1. Let $s \in \mathbb{R}, 0<p \leq \infty, 0<q \leq \infty$, and $\left\{\varphi_{j}\right\}_{j}$ a smooth dyadic resolution of unity. The Besov space $B_{p, q}^{s}\left(\mathbb{R}^{n}\right)$ is the set of all distributions $f \in \mathcal{S}^{\prime}\left(\mathbb{R}^{n}\right)$ such that

$$
\left\|f \mid B_{p, q}^{s}\left(\mathbb{R}^{n}\right)\right\|=\left(\sum_{j=0}^{\infty} 2^{j s q}\left\|\left(\varphi_{j} \hat{f}\right)^{\sim} \mid L_{p}\left(\mathbb{R}^{n}\right)\right\|^{q}\right)^{1 / q}
$$

is finite (with the usual modification if $q=\infty$ ).

Remark 1.2. The spaces $B_{p, q}^{s}\left(\mathbb{R}^{n}\right)$ are independent of the particular choice of the smooth dyadic resolution of unity $\left\{\varphi_{j}\right\}_{j}$ appearing in their definition. They are quasi-Banach spaces (Banach spaces for $p, q \geq 1$ ), and $\mathcal{S}\left(\mathbb{R}^{n}\right) \hookrightarrow B_{p, q}^{s}\left(\mathbb{R}^{n}\right) \hookrightarrow \mathcal{S}^{\prime}\left(\mathbb{R}^{n}\right)$, where the first embedding is dense if $p<\infty$ and $q<\infty$. The theory of the spaces $B_{p, q}^{s}\left(\mathbb{R}^{n}\right)$ has been developed in detail in $[13,14]$ (and continued and extended in the more recent monographs $[16,17])$, but has a longer history already including many contributors; we do not further want to discuss this here.

Note that the spaces $B_{p, q}^{s}\left(\mathbb{R}^{n}\right)$ contain tempered distributions which can only be interpreted as regular distributions (functions) for sufficiently high smoothness. More precisely, we have

$$
\begin{aligned}
& B_{p, q}^{s}\left(\mathbb{R}^{n}\right) \subset L_{1}^{\text {loc }}\left(\mathbb{R}^{n}\right) \text { if, and only if, } \\
& \qquad \begin{cases}s>\sigma_{p}, & \text { for } 0<p \leq \infty, 0<q \leq \infty, \\
s=\sigma_{p}, & \text { for } 0<p \leq 1,0<q \leq 1, \\
s=\sigma_{p}, & \text { for } 1<p \leq \infty, 0<q \leq \min (p, 2),\end{cases}
\end{aligned}
$$

see [10, Thm. 3.3.2]. In particular, for $s<\sigma_{p}$ one cannot interpret $f \in B_{p, q}^{s}\left(\mathbb{R}^{n}\right)$ as a regular distribution in general.

\section{LOCAL MEANS AND ATOMIC DECOMPOSITIONS}

There are equivalent characterizations for the Besov spaces $B_{p, q}^{s}\left(\mathbb{R}^{n}\right)$ in terms of local means and atomic decompositions. We first sketch the approach via local means. For further details we refer to $[2,3,17]$ with forerunners in [14, sec. 2.5.3].

Let $B=\left\{y \in \mathbb{R}^{n}:|y|<1\right\}$ be the unit ball in $\mathbb{R}^{n}$ and let $\kappa$ be a $C^{\infty}$ function in $\mathbb{R}^{n}$ with $\operatorname{supp} \kappa \subset B$. Then

$$
k(t, f)(x)=\int_{\mathbb{R}^{n}} \kappa(y) f(x+t y) \mathrm{d} y=t^{-n} \int_{\mathbb{R}^{n}} \kappa\left(\frac{y-x}{t}\right) f(y) \mathrm{d} y
$$

with $x \in \mathbb{R}^{n}$, and $t>0$ are local means (appropriately interpreted for $f \in S^{\prime}\left(\mathbb{R}^{n}\right)$ ). For given $s \in \mathbb{R}$ it is assumed that the kernel $\kappa$ satisfies in addition for some $\varepsilon>0$,

$$
\kappa^{\iota}(\xi) \neq 0 \text { if } 0<|\xi|<\varepsilon \quad \text { and } \quad\left(\mathrm{D}^{\alpha} \kappa^{\llcorner}\right)(0)=0 \text { if }|\alpha| \leq s .
$$


The second condition is empty if $s<0$. Furthermore, let $\kappa_{0}$ be a second $C^{\infty}$ function in $\mathbb{R}^{n}$ with $\operatorname{supp} \kappa_{0} \subset B$ and $\kappa_{0}^{\vee}(0) \neq 0$. The meaning of $k_{0}(f, t)$ is defined in the same way as (4) with $\kappa_{0}$ instead of $\kappa$.

We have the following characterization in terms of local means, see [17, Thm. 1.10] and $[9]$.

Theorem 1.3. Let $s \in \mathbb{R}$ and $0<q \leq \infty$. Let $\kappa_{0}$ and $\kappa$ be the above kernels of local means. Then, for $f \in S^{\prime}\left(\mathbb{R}^{n}\right)$,

$$
\left\|k_{0}(1, f) \mid L_{p}\left(\mathbb{R}^{n}\right)\right\|+\left(\sum_{j=1}^{\infty} 2^{j s q}\left\|k\left(2^{-j}, f\right) \mid L_{p}\left(\mathbb{R}^{n}\right)\right\|^{q}\right)^{1 / q}
$$

is an equivalent quasi-norm in $B_{p, q}^{s}\left(\mathbb{R}^{n}\right)$.

Remark 1.4. We shall only need one part of Theorem 1.3 , namely that $\left\|f \mid B_{p, q}^{s}\left(\mathbb{R}^{n}\right)\right\|$ can be estimated from below by (6). In that case some of the assumptions in (5) may be omitted. The inspection of the proof, see [9, Rem. 3], shows that if $\kappa$ is a $C^{\infty}$ function in $\mathbb{R}^{n}$ with

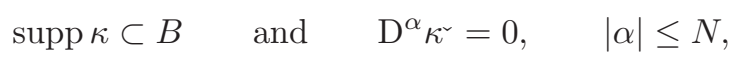

where $N>s-1$, then

$$
\left\|f \mid B_{p, q}^{s}\left(\mathbb{R}^{n}\right)\right\| \geq c\left(\sum_{j=1}^{\infty} 2^{j s q}\left\|k\left(2^{-j}, f\right) \mid L_{p}\left(\mathbb{R}^{n}\right)\right\|^{q}\right)^{1 / q}
$$

for some $c>0$.

The following atomic characterization closely follows the presentation in [15, sec. 13]. We introduce the relevant sequence spaces.

Definition 1.5. Let $0<p \leq \infty, 0<q \leq \infty$, and $\lambda=\left\{\lambda_{\nu, m} \in \mathbb{C}: \nu \in \mathbb{N}_{0}, m \in \mathbb{Z}^{n}\right\}$. Then

$$
b_{p, q}=\left\{\lambda:\left\|\lambda \mid b_{p, q}\right\|=\left(\sum_{\nu=0}^{\infty}\left(\sum_{m \in \mathbb{Z}^{n}}\left|\lambda_{\nu, m}\right|^{p}\right)^{q / p}\right)^{1 / q}<\infty\right\}
$$

(with the usual modification if $p=\infty$ and/or $q=\infty$ ).

The atoms we need are defined below.

\section{Definition 1.6.}

(i) Let $K \in \mathbb{N}_{0}$ and $d>1$. A $K$-times differentiable complex-valued function $a$ on $\mathbb{R}^{n}$ (continuous if $K=0$ ) is called a $1_{K}$-atom if

$$
\text { supp } a \subset d Q_{0, m} \text { for some } m \in \mathbb{Z}^{n},
$$

and

$$
\left|\mathrm{D}^{\alpha} a(x)\right| \leq 1 \quad \text { for } \quad|\alpha| \leq K
$$


(ii) Let $s \in \mathbb{R}, 0<p \leq \infty, K \in \mathbb{N}_{0}, L+1 \in \mathbb{N}_{0}$, and $d>1$. A $K$-times differentiable complex-valued function $a$ on $\mathbb{R}^{n}$ (continuous if $K=0$ ) is called

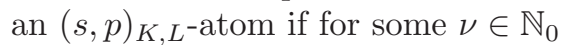

$$
\begin{gathered}
\operatorname{supp} a \subset d Q_{\nu, m} \quad \text { for some } \quad m \in \mathbb{Z}^{n}, \\
\left|\mathrm{D}^{\alpha} a(x)\right| \leq 2^{-\nu\left(s-\frac{n}{p}\right)+|\alpha| \nu} \quad \text { for }|\alpha| \leq K,
\end{gathered}
$$

and

$$
\int_{\mathbb{R}^{n}} x^{\beta} a(x) \mathrm{d} x=0 \quad \text { if }|\beta| \leq L .
$$

It is convenient to write $a_{\nu, m}(x)$ instead of $a(x)$ if this atom is located at $Q_{\nu, m}$ according to (7) and (8). Assumption (10) is called a moment condition, where $L=-1$ means that there are no moment conditions. Furthermore, $K$ denotes the smoothness of the atom, see (9). The atomic characterization of function spaces of type $B_{p, q}^{s}\left(\mathbb{R}^{n}\right)$ is given by the following result, see [15, Thm. 13.8].

Theorem 1.7. Let $0<p \leq \infty, 0<q \leq \infty$, and $s \in \mathbb{R}$. Let $d>1, K \in \mathbb{N}_{0}$, and $L+1 \in \mathbb{N}_{0}$ with

$$
K \geq(1+[s])_{+} \quad \text { and } \quad L \geq \max \left(-1,\left[\sigma_{p}-s\right]\right)
$$

be fixed. Then $f \in S^{\prime}\left(\mathbb{R}^{n}\right)$ belongs to $B_{p, q}^{s}\left(\mathbb{R}^{n}\right)$ if, and only if, it can be represented as

$$
f=\sum_{\nu=0}^{\infty} \sum_{m \in \mathbb{Z}^{n}} \lambda_{\nu, m} a_{\nu, m}(x), \quad \text { convergence being in } S^{\prime}\left(\mathbb{R}^{n}\right),
$$

where the $a_{\nu, m}$ are $1_{K}$-atoms $(\nu=0)$ or $(s, p)_{K, L}$-atoms $(\nu \in \mathbb{N})$ with

$$
\operatorname{supp} a_{\nu, m} \subset d Q_{\nu, m}, \quad \nu \in \mathbb{N}_{0}, \quad m \in \mathbb{Z}^{n},
$$

and $\lambda \in b_{p, q}$. Furthermore,

$$
\inf \left\|\lambda \mid b_{p, q}\right\|,
$$

where the infimum is taken over all admissible representations (11), is an equivalent quasi-norm in $B_{p, q}^{s}\left(\mathbb{R}^{n}\right)$.

\section{Dilation operators}

We now state the main result.

Theorem 2.1. Let $0<p \leq 1,0<q \leq \infty, k \in \mathbb{N}$, and $T_{k}$ be defined by (1). Then

$$
\left\|T_{k} \mid \mathcal{L}\left(B_{p, q}^{\sigma_{p}}\left(\mathbb{R}^{n}\right)\right)\right\| \sim 2^{k\left(\sigma_{p}-\frac{n}{p}\right)} k^{1 / q}=2^{-k n} k^{1 / q},
$$

where the constants of equivalence do not depend on $k$. 
Proof. For convenience we assume $q<\infty$ in the sequel, but the counterpart for $q=\infty$ is obvious.

- Step 1. We give an estimate for the upper bounds of the dilation operators $T_{k}$ similar to [18, Prop. 3.2]. Since the techniques used there even fail for $p=1$, we need to find suitable substitutes when $0<p \leq 1$.

Recall Definition 1.1, where in particular the dyadic resolution of unity was constructed such that

$$
\varphi_{j}(x)=\varphi\left(2^{-j} x\right)-\varphi\left(2^{-j+1} x\right), \quad j \in \mathbb{N} .
$$

Elementary calculation yields

$$
\left(\varphi_{j}(\xi) \widehat{f\left(2^{k} \cdot\right)}(\xi)\right)^{\swarrow}(x)=2^{-k n}\left(\varphi_{j}(\xi) \hat{f}\left(2^{-k} \xi\right)\right)^{\swarrow}(x)=\left(\varphi_{j}\left(2^{k} \xi\right) \hat{f}(\xi)\right)^{\swarrow}\left(2^{k} x\right) .
$$

From the definition of Besov spaces with $f\left(2^{k} x\right)$ in place of $f(x)$ we obtain

$$
\begin{aligned}
\left\|f\left(2^{k} \cdot\right) \mid B_{p, q}^{\sigma_{p}}\right\| & =\left(\sum_{j=0}^{\infty} 2^{j \sigma_{p} q}\left\|\left(\varphi_{j}\left(2^{k} \cdot\right) \hat{f}\right)^{\varsigma}\left(2^{k} \cdot\right) \mid L_{p}\right\|^{q}\right)^{1 / q} \\
& =2^{-k \frac{n}{p}}\left(\sum_{j=0}^{\infty} 2^{j \sigma_{p} q}\left\|\left(\varphi_{j}\left(2^{k} \cdot\right) \hat{f}\right)^{\llcorner} \mid L_{p}\right\|^{q}\right)^{1 / q} .
\end{aligned}
$$

If $j \geq k+1$, then $\varphi_{j}\left(2^{k} x\right)=\varphi_{j-k}(x)$. This gives

$$
\begin{aligned}
2^{-k \frac{n}{p}} & \left(\sum_{j=k+1}^{\infty} 2^{j \sigma_{p} q}\left\|\left(\varphi_{j}\left(2^{k} \cdot\right) \hat{f}\right)^{\swarrow} \mid L_{p}\right\|^{q}\right)^{1 / q} \\
& =2^{-k \frac{n}{p}}\left(\sum_{j=k+1}^{\infty} 2^{(j-k) \sigma_{p} q} 2^{k \sigma_{p} q}\left\|\left(\varphi_{j-k} \hat{f}\right)^{\llcorner} \mid L_{p}\right\|^{q}\right)^{1 / q} \\
& =2^{-k \frac{n}{p}+k \sigma_{p}}\left(\sum_{l=1}^{\infty} 2^{l \sigma_{p} q}\left\|\left(\varphi_{l} \hat{f}\right)^{\curlyvee} \mid L_{p}\right\|^{q}\right)^{1 / q} \\
& \leq c 2^{-k n}\left\|f \mid B_{p, q}^{\sigma_{p}}\right\| .
\end{aligned}
$$

For the further calculations we make use of a Fourier multiplier theorem from [13, Prop. 1.5.1]. We have

$$
\left\|(M \hat{h})^{\llcorner}\left|L_{p}\|\leq c\| M^{\curvearrowright}\right| L_{p}\right\| \cdot\left\|h \mid L_{p}\right\| \quad \text { if } \quad 0<p \leq 1,
$$

with $M^{\ulcorner} \in S^{\prime} \cap L_{p}$, and supp $\hat{h} \subset \Omega$, supp $M \subset \Gamma$, where $\Omega$ and $\Gamma$ are compact subsets of $\mathbb{R}^{n}$. ( $c$ does not depend on $M$ and $h$, but may depend on $\Omega$ and $\Gamma$.)

Of course, for $p=1$, this is just the Hausdorff-Young inequality (which was also used in [18]). 
We put $h=\left(\varphi_{0} \hat{f}\right)^{\sim}$, where $\operatorname{supp} \hat{h} \subset \operatorname{supp} \varphi_{0}=\Omega$. If $j=0$, we take $M_{0}=\varphi_{0}\left(2^{k} \cdot\right)$, where $\operatorname{supp} M_{0} \subset \operatorname{supp} \varphi_{0}=\Gamma$, and calculate

$$
\begin{aligned}
& 2^{-k \frac{n}{p}}\left\|\left(\varphi_{0}\left(2^{k} \cdot\right) \hat{f}\right)^{\swarrow}\left|L_{p}\left\|\leq c 2^{-k \frac{n}{p}}\right\| \varphi_{0}\left(2^{k} \cdot\right)^{\swarrow}\right| L_{p}\right\| \cdot\left\|\left(\varphi_{0} \hat{f}\right)^{\swarrow} \mid L_{p}\right\| \\
& =c 2^{-k \frac{n}{p}} 2^{k \sigma_{p}}\left\|\varphi_{0^{\sim}}\left|L_{p}\|\cdot\|\left(\varphi_{0} \hat{f}\right)^{\sim}\right| L_{p}\right\| \\
& =c^{\prime} 2^{k\left(\sigma_{p}-\frac{n}{p}\right)}\left\|\left(\varphi_{0} \hat{f}\right)^{`} \mid L_{p}\right\| \\
& \leq c^{\prime} 2^{k\left(\sigma_{p}-\frac{n}{p}\right)}\left\|f \mid B_{p, q}^{\sigma_{p}}\right\| \\
& =c^{\prime} 2^{-k n}\left\|f \mid B_{p, q}^{\sigma_{p}}\right\| \text {. }
\end{aligned}
$$

Finally it remains to consider $1 \leq j \leq k$. This is the crucial step leading to $k^{1 / q}$. In this case $\varphi_{j}(x)=\bar{\varphi}\left(2^{-j} x\right)$, where $\bar{\varphi}=\varphi(x)-\varphi(2 x)$. Hence

$$
\begin{aligned}
2^{-k \frac{n}{p}}\left(\sum_{j=1}^{k} 2^{j \sigma_{p} q}\left\|\left(\varphi_{j}\left(2^{k} \cdot\right) \hat{f}\right)^{\swarrow} \mid L_{p}\right\|^{q}\right)^{1 / q} \\
\quad=2^{-k \frac{n}{p}}\left(\sum_{j=1}^{k} 2^{j \sigma_{p} q}\left\|\left(\bar{\varphi}\left(2^{k-j} \cdot\right) \hat{f}\right)^{\llcorner} \mid L_{p}\right\|^{q}\right)^{1 / q} \\
\quad=2^{-k \frac{n}{p}}\left(\sum_{j=1}^{k-1} 2^{j \sigma_{p} q}\left\|\left(\bar{\varphi}\left(2^{k-j} \cdot\right) \varphi_{0} \hat{f}\right)^{\llcorner}\left|L_{p}\left\|^{q}+2^{k \sigma_{p} q}\right\|(\bar{\varphi} \hat{f})^{\llcorner}\right| L_{p}\right\|^{q}\right)^{1 / q} .
\end{aligned}
$$

The term in (16) originating from $j=k$ needs some extra care. Using (14) where we set $M=\varphi_{0}(2 \cdot), \operatorname{supp} M \subset \operatorname{supp} \varphi_{0}=\Gamma$, we obtain

$$
\begin{aligned}
& 2^{k \sigma_{p} q}\left\|(\bar{\varphi} \hat{f})^{\llcorner}\left|L_{p}\left\|^{q}=2^{k \sigma_{p} q}\right\|\left(\varphi_{0} \hat{f}\right)^{\swarrow}-\left(\varphi_{0}(2 \cdot) \hat{f}\right)^{\llcorner}\right| L_{p}\right\|^{q} \\
& \leq c 2^{k \sigma_{p} q}\left(\left\|\left(\varphi_{0} \hat{f}\right)^{`}\left|L_{p}\|+\|\left(\varphi_{0}(2 \cdot) \varphi_{0} \hat{f}\right)^{`}\right| L_{p}\right\|\right)^{q} \\
& \leq c^{\prime} 2^{k \sigma_{p} q}\left\|\left(\varphi_{0} \hat{f}\right)^{`} \mid L_{p}\right\|^{q}\left(1+\left\|\varphi_{0}{ }^{\curvearrowright}(2 \cdot) \mid L_{p}\right\|\right)^{q} \\
& =c_{1} 2^{k \sigma_{p} q}\left\|\left(\varphi_{0} \hat{f}\right)^{\sim} \mid L_{p}\right\|^{q} \text {. }
\end{aligned}
$$

This estimate can be incorporated into our further calculations. For $1 \leq j \leq k-1$ we use the multiplier theorem with $M_{j}=\bar{\varphi}\left(2^{k-j}.\right)$, and observe that

$$
\operatorname{supp} M_{j} \subset\left\{x:\left|2^{k-j} x\right| \leq 2\right\} \subset\{x:|x| \leq 2\}=\Gamma .
$$


Now (16) yields

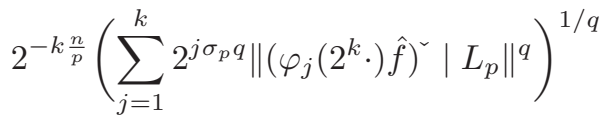

$$
\begin{aligned}
& \leq c 2^{-k \frac{n}{p}}\left(\sum_{j=1}^{k-1} 2^{j \sigma_{p} q}\left\|\left(\bar{\varphi}\left(2^{k-j} \cdot\right)\right)^{\swarrow}\left|L_{p}\left\|^{q} \cdot\right\|\left(\varphi_{0} \hat{f}\right)^{\swarrow}\right| L_{p}\right\|^{q}\right. \\
& \left.+2^{k \sigma_{p} q}\left\|\left(\varphi_{0} \hat{f}\right)^{\smile} \mid L_{p}\right\|^{q}\right)^{1 / q} \\
& =c_{2} 2^{-k \frac{n}{p}} \cdot\left\|\left(\varphi_{0} \hat{f}\right)^{\swarrow} \mid L_{p}\right\|\left(\sum_{j=1}^{k-1} 2^{j \sigma_{p} q}\left\|\left(\bar{\varphi}\left(2^{k-j} \cdot\right)\right)^{\swarrow} \mid L_{p}\right\|^{q}+2^{k \sigma_{p} q}\right)^{1 / q} \\
& \leq c_{2} 2^{-k \frac{n}{p}} \cdot\left\|f \mid B_{p q}^{\sigma_{p}}\right\|\left(\sum_{j=1}^{k-1} 2^{j \sigma_{p} q}\left\|\left(\bar{\varphi}\left(2^{k-j} \cdot\right)\right)^{\llcorner} \mid L_{p}\right\|^{q}+2^{k \sigma_{p} q}\right)^{1 / q} \\
& \leq c_{2} 2^{-k \frac{n}{p}} \cdot\left\|f \mid B_{p q}^{\sigma_{p}}\right\|\left(\sum_{j=1}^{k-1} 2^{j\left(\frac{n}{p}-n\right) q}\left\|2^{(j-k) n} \bar{\varphi}^{\curlyvee}\left(2^{j-k} \cdot\right) \mid L_{p}\right\|^{q}+2^{k \sigma_{p} q}\right)^{1 / q} \\
& \leq c_{2} 2^{-k \frac{n}{p}} \cdot\left\|f \mid B_{p q}^{\sigma_{p}}\right\| \\
& \times\left(\sum_{j=1}^{k-1} 2^{j\left(\frac{n}{p}-n\right) q} 2^{(j-k) n q} 2^{-(j-k) n \cdot \frac{1}{p} \cdot q}\left\|\bar{\varphi}^{\triangleleft} \mid L_{p}\right\|^{q}+2^{k \sigma_{p} q}\right)^{1 / q} \\
& \leq c_{3} 2^{-k \frac{n}{p}} \cdot\left\|f \mid B_{p q}^{\sigma_{p}}\right\|\left(\sum_{j=1}^{k} 2^{k \sigma_{p} q}\right)^{1 / q} \\
& =c_{3} k^{1 / q} 2^{-k n} \cdot\left\|f \mid B_{p q}^{\sigma_{p}}\right\| .
\end{aligned}
$$

Finally (12) together with (13), (15), and (17) give the upper estimate.

- Step 2. It remains to prove that the estimate is sharp. Let $\psi \in S\left(\mathbb{R}^{n}\right)$ be a non-negative function with support in $\left\{x \in \mathbb{R}^{n}:|x| \leq 1 / 8\right\}$ and $\int_{\mathbb{R}^{n}} \psi(x) \mathrm{d} x=1$. We show that, for $0<q \leq \infty$,

$$
\left\|\psi\left(2^{k} \cdot\right) \mid B_{p, q}^{\sigma_{p}}\right\| \geq c 2^{-k n} k^{1 / q}, \quad k \in \mathbb{N} .
$$

Let us take a function $\kappa \in S\left(\mathbb{R}^{n}\right)$ with

$$
\left(\mathrm{D}^{\alpha} \kappa^{\sim}\right)(0)=0, \quad|\alpha| \leq r,
$$

where $r>\sigma_{p}-1$. According to remark 1.4 these conditions on $\kappa$ are sufficient for our purposes. Furthermore, we require

$$
\kappa(x)=1, \quad \text { if } \quad x \in M=\left\{z \in \mathbb{R}^{n}:|z-(1 / 2,0, \ldots, 0)|<1 / 4\right\} .
$$


We construct a function $\kappa$ that satisfies (18) and (19). Let us first consider the one-dimensional case $n=1$. Put

$$
f(x)=\frac{\mathrm{d}^{r}}{\mathrm{~d} x^{r}} f_{0}(x), \quad \text { where } \quad f_{0} \in S(\mathbb{R}) .
$$

Then we have that

$$
f^{\sim}(\xi)=-i^{r}\left(x^{r} f_{0}^{\sim}\right)(\xi)=-i^{r} \xi^{r} f_{0}^{\sim}(\xi) .
$$

In particular, for $l<r$ we calculate

$$
\left(\frac{\mathrm{d}^{l}}{\mathrm{~d} x^{l}} f^{\sim}\right)(0)=-\left.i^{r} \sum_{j=0}^{l}\left(\begin{array}{l}
l \\
j
\end{array}\right) \frac{r !}{(r-j) !} \xi^{r-j} \frac{\mathrm{d}^{l-j}}{\mathrm{~d} x^{l-j}} f_{0}^{\sim}(\xi)\right|_{\xi=0}=0,
$$

from which we see that $f$ satisfies the moment conditions. Needing

$$
f(x)=1, \quad \text { for } \quad \frac{1}{4}<x<\frac{3}{4},
$$

we put $f_{0}(x):=x^{r} \cdot \beta(x)$, where $\beta \in S(\mathbb{R})$ is chosen such that

$$
\beta(x)=\frac{1}{r !}, \quad x \in B_{1}\left(\frac{1}{2}\right) .
$$

The previous considerations can easily be extended to higher dimensions by setting

$$
g\left(x_{1}, \ldots, x_{n}\right)=f_{0}\left(x_{1}\right) f_{0}\left(x_{2}-\frac{1}{2}\right) \cdots f_{0}\left(x_{n}-\frac{1}{2}\right),
$$

and finally

$$
\kappa(x)=\mathrm{D}^{(r, \ldots, r)} g(x), \quad x \in \mathbb{R}^{n},
$$

gives the desired function, if we choose $r>\sigma_{p}-1$.

When $j=1,2, \ldots, k$, we set $y_{0}=(1 / 2,0, \ldots, 0), x_{0}=-2^{-j} y_{0}$, and

$$
\omega=2^{k} x+2^{k-j} y=2^{k}\left(x_{0}+\tilde{x}\right)+2^{k-j}\left(y_{0}+\tilde{y}\right)=2^{k} \tilde{x}+2^{k-j} \tilde{y} .
$$

If $\omega \in \operatorname{supp} \psi$ and $|\tilde{x}| \leq \frac{1}{8} 2^{-j}$ we see that

$$
|\tilde{y}|=\left|2^{-k+j} \omega-2^{j} \tilde{x}\right| \leq 2^{-k+j} \frac{1}{8}+\frac{1}{8} \leq \frac{1}{4} .
$$

This yields for $\left|x-\left(-\frac{1}{2} \cdot \frac{1}{2^{j}}, 0, \ldots, 0\right)\right|<\frac{1}{2^{j}} \frac{1}{8}$ that

$$
\operatorname{supp}_{y} \psi\left(2^{k} x+2^{k-j} y\right) \subset M \text {. }
$$

For these $x$ we get

$$
\begin{aligned}
\mathcal{K}\left(2^{-j}, \psi\left(2^{k} \cdot\right)\right)(x) & =\int_{\mathbb{R}^{n}} \kappa(y) \psi\left(2^{k} x+2^{k-j} y\right) \mathrm{d} y \\
& =\int_{\mathbb{R}^{n}} \psi\left(2^{k} x+2^{k-j} y\right) \mathrm{d} y=2^{(j-k) n} .
\end{aligned}
$$


Hence,

$$
\left\|\mathcal{K}\left(2^{-j}, \psi\left(2^{k} \cdot\right)\right) \mid L_{p}\right\| \geq c 2^{-\frac{j n}{p}} 2^{(j-k) n}=c 2^{-j n\left(\frac{1}{p}-1\right)} 2^{-k n}=c 2^{-j \sigma_{p}} 2^{-k n} .
$$

This yields

$$
\begin{aligned}
\left\|\psi\left(2^{k} \cdot\right) \mid B_{p, q}^{\sigma_{p}}\right\| & \geq \bar{c}\left(\sum_{j=1}^{k} 2^{j \sigma_{p} q}\left\|\mathcal{K}\left(2^{-j}, \psi\left(2^{k} \cdot\right)\right) \mid L_{p}\right\|^{q}\right)^{1 / q} \\
& \geq c^{\prime} 2^{-k n}\left(\sum_{j=1}^{k} 1\right)^{1 / q}=c^{\prime} 2^{-k n} k^{1 / q}
\end{aligned}
$$

which is the desired result.

\section{Applications}

\subsection{Besov spaces with positive smoothness on $\mathbb{R}^{n}$}

With the help of the previous results on dilation operators, we want to discuss in this section the connection and diversity of three different approaches to Besov spaces with positive smoothness.

In addition to the Fourier-analytical approach, see Definition 1.1, we now present two further characterizations - associated to definitions by differences and subatomic decompositions - before we come to some comparison.

The Classical approach: Besov spaces $\mathbf{B}_{p, q}^{s}\left(\mathbb{R}^{n}\right)$

If $f$ is an arbitrary function on $\mathbb{R}^{n}, h \in \mathbb{R}^{n}$, and $k \in \mathbb{N}$, then

$$
\left(\Delta_{h}^{1} f\right)(x)=f(x+h)-f(x) \quad \text { and } \quad\left(\Delta_{h}^{k+1} f\right)(x)=\Delta_{h}^{1}\left(\Delta_{h}^{k} f\right)(x), \quad k \in \mathbb{N} .
$$

For convenience we may write $\Delta_{h}$ instead of $\Delta_{h}^{1}$. Furthermore, the $k$ th modulus of smoothness of a function $f \in L_{p}\left(\mathbb{R}^{n}\right), 0<p \leq \infty, k \in \mathbb{N}$, is defined by

$$
\omega_{k}(f, t)_{p}=\sup _{|h| \leq t}\left\|\Delta_{h}^{k} f \mid L_{p}\left(\mathbb{R}^{n}\right)\right\|, \quad t>0 .
$$

We shall simply write $\omega(f, t)_{p}$ instead of $\omega_{1}(f, t)_{p}$ and $\omega(f, t)$ instead of $\omega(f, t)_{\infty}$.

Definition 3.1. Let $0<p, q \leq \infty, s>0$, and $r \in \mathbb{N}$ such that $r>s$. Then the Besov space $\mathbf{B}_{p, q}^{s}\left(\mathbb{R}^{n}\right)$ contains all $f \in L_{p}\left(\mathbb{R}^{n}\right)$ such that

$$
\left\|f\left|\mathbf{B}_{p, q}^{s}\left(\mathbb{R}^{n}\right)\left\|_{r}=\right\| f\right| L_{p}\left(\mathbb{R}^{n}\right)\right\|+\left(\int_{0}^{1} t^{-s q} \omega_{r}(f, t)_{p}^{q} \frac{\mathrm{d} t}{t}\right)^{1 / q}
$$

(with the usual modification if $q=\infty$ ) is finite. 
Remark 3.2. These are the classical Besov spaces, in particular, when $1 \leq p, q \leq \infty$, $s>0$. The study for all admitted $s, p$, and $q$ goes back to [11], we also refer to $[1$, ch. 5, Def. 4.3$]$ and $[4$, ch. $2, \S 10]$. There are as well many older references in the literature devoted to the cases $p, q \geq 1$. A recent approach including atomic characterizations is given in [7] based on [8].

The spaces in Definition 3.1 are independent of $r$, meaning that different values of $r>s$ result in quasi-norms which are equivalent. Furthermore the spaces are quasi-Banach spaces (Banach spaces if $p, q \geq 1$ ). Note that we deal with subspaces of $L_{p}\left(\mathbb{R}^{n}\right)$, in particular we have the embedding

$$
\mathbf{B}_{p, q}^{s}\left(\mathbb{R}^{n}\right) \hookrightarrow L_{p}\left(\mathbb{R}^{n}\right), \quad s>0, \quad 0<q \leq \infty, \quad 0<p \leq \infty .
$$

The classical scale of Besov spaces contains many well-known function spaces. For example, if $p=q=\infty$, one recovers the Hölder-Zygmund spaces $\mathcal{C}^{s}\left(\mathbb{R}^{n}\right)$,

$$
\mathbf{B}_{\infty, \infty}^{s}\left(\mathbb{R}^{n}\right)=\mathcal{C}^{s}\left(\mathbb{R}^{n}\right), \quad s>0 .
$$

We add the following homogeneity estimate, which will serve us later on. Let $R>0$, $s>0$, and $0<p, q \leq \infty$. Then

$$
\left\|f(R \cdot)\left|\mathbf{B}_{p, q}^{s}\left(\mathbb{R}^{n}\right)\left\|\leq c \max \left(R^{-\frac{n}{p}}, R^{s-\frac{n}{p}}\right)\right\| f\right| \mathbf{B}_{p, q}^{s}\left(\mathbb{R}^{n}\right)\right\| .
$$

To prove this we simply observe that

$$
\begin{aligned}
\left\|f(R \cdot) \mid \mathbf{B}_{p, q}^{s}\left(\mathbb{R}^{n}\right)\right\| & =\left\|f(R \cdot) \mid L_{p}\left(\mathbb{R}^{n}\right)\right\|+\left(\int_{0}^{1} t^{-s q} \omega_{r}(f(R \cdot), t)_{p}^{q} \frac{\mathrm{d} t}{t}\right)^{1 / q} \\
& =R^{-\frac{n}{p}}\left\|f \mid L_{p}\left(\mathbb{R}^{n}\right)\right\|+R^{-\frac{n}{p}}\left(\int_{0}^{1} t^{-s q} \omega_{r}(f, R t)_{p}^{q} \frac{\mathrm{d} t}{t}\right)^{1 / q} \\
& =R^{-\frac{n}{p}}\left\|f \mid L_{p}\left(\mathbb{R}^{n}\right)\right\|+R^{s-\frac{n}{p}}\left(\int_{0}^{R} \tau^{-s q} \omega_{r}(f, \tau)_{p}^{q} \frac{\mathrm{d} \tau}{\tau}\right)^{1 / q} \\
& \leq c \max \left(R^{-\frac{n}{p}}, R^{s-\frac{n}{p}}\right)\left\|f \mid \mathbf{B}_{p, q}^{s}\left(\mathbb{R}^{n}\right)\right\| .
\end{aligned}
$$

Here we used that the integration over $t \in(0,1)$ in $(20)$ can be replaced by an integration over $t \in(0, \infty)$.

The subatomic approach: Besov spaces $\mathfrak{B}_{p, q}^{s}\left(\mathbb{R}^{n}\right)$

The subatomic approach provides a constructive definition for Besov spaces, expanding functions $f$ via building blocks and suitable coefficients, where the latter belong to certain sequence spaces. For further details on the subject we refer to $[6,17]$. Let

$$
\mathbb{R}_{++}^{n}:=\left\{y \in \mathbb{R}^{n}: y=\left(y_{1}, \ldots, y_{n}\right), y_{j}>0\right\} .
$$


Definition 3.3. Let $k$ be a non-negative $C^{\infty}$ function in $\mathbb{R}^{n}$ with

$$
\operatorname{supp} k \subset\left\{y \in \mathbb{R}^{n}:|y|<2^{J-\varepsilon}\right\} \cap \mathbb{R}_{++}^{n}
$$

for some fixed $\varepsilon>0$ and some fixed $J \in \mathbb{N}$, satisfying

$$
\sum_{m \in \mathbb{Z}^{n}} k(x-m)=1, \quad x \in \mathbb{R}^{n} .
$$

Let $\beta \in \mathbb{N}_{0}^{n}, j \in \mathbb{N}_{0}, m \in \mathbb{Z}^{n}$, and let $k^{\beta}(x)=\left(2^{-J} x\right)^{\beta} k(x)$. Then

$$
k_{j, m}^{\beta}(x)=k^{\beta}\left(2^{j} x-m\right)
$$

denote the building blocks related to $Q_{j, m}$.

We consider the following sequence spaces.

Definition 3.4. Let $\varrho \geq 0, s \in \mathbb{R}, 0<p, q \leq \infty$, and

$$
\lambda=\left\{\lambda_{j, m}^{\beta} \in \mathbb{C}: \beta \in \mathbb{N}_{0}^{n}, m \in \mathbb{Z}^{n}, j \in \mathbb{N}_{0}\right\} .
$$

Then the sequence space $b_{p, q}^{s, \varrho}$ is defined as

$$
b_{p, q}^{s, \varrho}:=\left\{\lambda:\left\|\lambda \mid b_{p, q}^{s, \varrho}\right\|<\infty\right\}
$$

where

$$
\left\|\lambda \mid b_{p, q}^{s, \varrho}\right\|=\sup _{\beta \in \mathbb{N}_{0}^{n}} 2^{\varrho|\beta|}\left(\sum_{j=0}^{\infty} 2^{j(s-n / p) q}\left(\sum_{m \in \mathbb{Z}^{n}}\left|\lambda_{j, m}^{\beta}\right|^{p}\right)^{q / p}\right)^{1 / q}
$$

(with the usual modification if $p=\infty$ and/or $q=\infty$ ).

Remark 3.5. It might not be obvious immediately, but the building blocks $k_{j, m}^{\beta}$ in our subatomic approach differ from the atoms $a$ - used to characterize the spaces $B_{p, q}^{s}\left(\mathbb{R}^{n}\right)$ in Theorem 1.7 - mainly by the imposed moment conditions on the latter and some unimportant technicalities. In particular, the normalizing factors $2^{\nu\left(s-\frac{n}{p}\right)}$ are incorporated in the sequence spaces $b_{p, q}^{s, \varrho}$ in the subatomic approach; recall Definition 1.5.

Definition 3.6. Let $s>0,0<p \leq \infty, 0<q \leq \infty, \varrho \geq 0$. Then $\mathfrak{B}_{p, q}^{s}\left(\mathbb{R}^{n}\right)$ contains all $f \in L_{p}\left(\mathbb{R}^{n}\right)$ which can be represented as

$$
f(x)=\sum_{\beta \in \mathbb{N}_{0}^{n}} \sum_{j=0}^{\infty} \sum_{m \in \mathbb{Z}^{n}} \lambda_{j, m}^{\beta} k_{j, m}^{\beta}(x), \quad x \in \mathbb{R}^{n},
$$

with coefficients $\lambda=\left\{\lambda_{j, m}^{\beta}\right\}_{\beta \in \mathbb{N}_{0}^{n}, j \in \mathbb{N}_{0}, m \in \mathbb{Z}^{n}} \in b_{p, q}^{s, \varrho}$, and equipped with the quasi-norm

$$
\left\|f\left|\mathfrak{B}_{p, q}^{s}\left(\mathbb{R}^{n}\right)\|=\inf \| \lambda\right| b_{p, q}^{s, \varrho}\right\|
$$

where the infimum is taken over all possible representations (22). 
Remark 3.7. The definitions given above follow closely [17, sec. 9.2]. The spaces $\mathfrak{B}_{p, q}^{s}\left(\mathbb{R}^{n}\right)$ are quasi-Banach spaces (Banach spaces for $p, q \geq 1$ ) and independent of $k$ and $\varrho$ (in terms of equivalent quasi-norms). Furthermore, we have the embedding

$$
\mathfrak{B}_{p, q}^{s}\left(\mathbb{R}^{n}\right) \longleftrightarrow L_{p}\left(\mathbb{R}^{n}\right), \quad 0<p \leq \infty
$$

see [17, Thm. 9.8]. Concerning the convergence of (22) one obtains as a consequence of $\lambda \in b_{p, q}^{s, \varrho}$ that the series on the right-hand side converges absolutely in $L_{p}\left(\mathbb{R}^{n}\right)$ if $p<\infty$, and in $L_{\infty}\left(\mathbb{R}^{n}, w_{\sigma}\right)$ if $p=\infty$, where $w_{\sigma}(x)=\left(1+|x|^{2}\right)^{\sigma / 2}$ with $\sigma<0$. Since this implies unconditional convergence we may simplify (22) and write in the sequel

$$
f=\sum_{\beta, j, m} \lambda_{j, m}^{\beta} k_{j, m}^{\beta} .
$$

\section{Connections And DIVERsity}

We now discuss the coincidence and diversity of the above presented concepts of Besov spaces and may restrict ourselves to positive smoothness $s>0$. In view of our Remarks 1.2, 3.2, and 3.7 concerning the different nature of these spaces, it is obvious that there cannot be established a complete coincidence of all approaches when $s<\sigma_{p}$, since $\mathbf{B}_{p, q}^{s}\left(\mathbb{R}^{n}\right)$ and $\mathfrak{B}_{p, q}^{s}\left(\mathbb{R}^{n}\right)$ are always subspaces of $L_{p}\left(\mathbb{R}^{n}\right)$ and thus contain functions, whereas the elements of $B_{p, q}^{s}\left(\mathbb{R}^{n}\right)$ are distributions which can be interpreted as regular distributions ('functions') if, and only if, (3) is satisfied. However, when $s>\sigma_{p}$, the outcome is optimal in the sense that all approaches result in the same Besov space.

Theorem 3.8. Let $s>0,0<p \leq \infty, 0<q \leq \infty$.

(i) Then

$$
\mathbf{B}_{p, q}^{s}\left(\mathbb{R}^{n}\right)=\mathfrak{B}_{p, q}^{s}\left(\mathbb{R}^{n}\right)
$$

(in the sense of equivalent quasi-norms) as subsets of $L_{p}\left(\mathbb{R}^{n}\right)$.

(ii) Let $s>\sigma_{p}$, then

$$
B_{p, q}^{s}\left(\mathbb{R}^{n}\right)=\mathbf{B}_{p, q}^{s}\left(\mathbb{R}^{n}\right)=\mathfrak{B}_{p, q}^{s}\left(\mathbb{R}^{n}\right)
$$

(in the sense of equivalent quasi-norms) in $S^{\prime}\left(\mathbb{R}^{n}\right)$. 


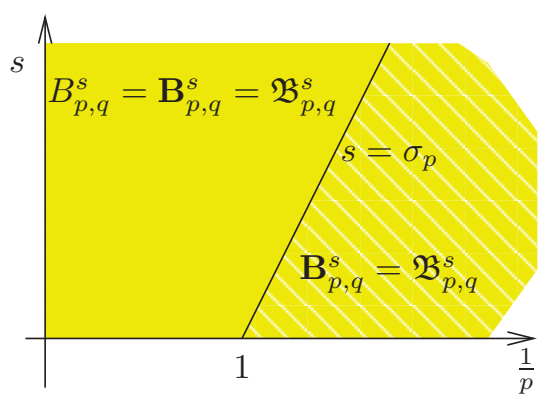

Remark 3.9. The first equality in (24) is longer known, see [13, Thm. 2.5.12; 14, Thm. 2.6.1] with forerunners in case of $p, q \geq 1$, see [12, 2.5.1, 2.7.2], whereas the second equality in (24) is a consequence of the recently proved coincidence (23), see [17, Prop. 9.14] (with forerunners in [15, sec. 14.15; 16, Thm. 2.9]). It essentially relies on the atomic decomposition, see $[7, \mathrm{Thm} .1 .1 .14 ; 8]$. In the figure we have indicated the situation in the usual $\left(\frac{1}{p}, s\right)$-diagram.

Our results on the norms of the dilation operators $T_{k}$ established in Theorem 2.1 now lead to new insights when dealing with the limiting case $s=\sigma_{p}$. By (3) the spaces $B_{p, q}^{\sigma_{p}}\left(\mathbb{R}^{n}\right)$ and $\mathbf{B}_{p, q}^{\sigma_{p}}\left(\mathbb{R}^{n}\right)$ with $0<p<1$ and $q>1$ are incomparable. If $q \leq 1$ the spaces can be compared. We obtain the following assertion.

Corollary 3.10. Let $0<p<1$, and $0<q \leq 1$. Then

$$
B_{p, q}^{\sigma_{p}}\left(\mathbb{R}^{n}\right) \neq \mathbf{B}_{p, q}^{\sigma_{p}}\left(\mathbb{R}^{n}\right)
$$

(in terms of equivalent quasi-norms) as sets of measurable functions.

Proof. We use the homogeneity estimate (21), which for $R=2^{k}, s>0$, and $0<p, q \leq \infty$ reads

$$
\left\|f\left(2^{k} \cdot\right)\left|\mathbf{B}_{p, q}^{s}\left\|\leq c 2^{k\left(s-\frac{n}{p}\right)}\right\| f\right| \mathbf{B}_{p, q}^{s}\right\| .
$$

We proceed indirectly, assuming that $B_{p, q}^{\sigma_{p}}\left(\mathbb{R}^{n}\right)=\mathbf{B}_{p, q}^{\sigma_{p}}\left(\mathbb{R}^{n}\right)$ for $0<q \leq 1$. But then using Theorem 2.1 and (25) above, we could find a function $\psi \in B_{p, q}^{\sigma_{p}}$ with

$$
\begin{aligned}
2^{k\left(\sigma_{p}-\frac{n}{p}\right)} k^{1 / q}\left\|\psi \mid B_{p, q}^{\sigma_{p}}\right\| & \leq c\left\|\psi\left(2^{k} \cdot\right)\left|B_{p, q}^{\sigma_{p}}\|\sim\| \psi\left(2^{k} \cdot\right)\right| \mathbf{B}_{p, q}^{\sigma_{p}}\right\| \\
& \leq c 2^{k\left(\sigma_{p}-\frac{n}{p}\right)}\left\|\psi \mid \mathbf{B}_{p, q}^{\sigma_{p}}\right\| \\
& \sim c 2^{k\left(\sigma_{p}-\frac{n}{p}\right)}\left\|\psi \mid B_{p, q}^{\sigma_{p}}\right\|,
\end{aligned}
$$

leading to

$$
k^{1 / q} \leq c, \quad k \in \mathbb{N} .
$$

This gives the desired contradiction. 


\subsection{A comment on atomic expansion}

Following [18, Rem. 3.7] one can show that first moment conditions on the line $s=\sigma_{p}$ are indeed necessary. This immediately leads to

$$
B_{p, q}^{\sigma_{p}}\left(\mathbb{R}^{n}\right) \neq \mathfrak{B}_{p, q}^{\sigma_{p}}\left(\mathbb{R}^{n}\right), \quad 0<p<1, \quad 0<q<\infty,
$$

in view of Remark 3.5. We sketch the proof.

Every $f \in B_{p, q}^{\sigma_{p}}\left(\mathbb{R}^{n}\right)$ may be represented by optimal atomic decompositions

$$
f(x)=\sum_{\nu, m} \lambda_{\nu, m} a_{\nu, m}(x), \quad x \in \mathbb{R}^{n},
$$

with

$$
\left\|\lambda\left|b_{p, q}\|\leq c\| f\right| B_{p, q}^{\sigma_{p}}\right\|, \quad f \in B_{p, q}^{\sigma_{p}}\left(\mathbb{R}^{n}\right),
$$

see $[17$, ch. 1.5] for details. If no moment conditions were required here, then

$$
g_{k}(x)=f\left(2^{k} x\right)=\sum_{\nu, m} \lambda_{\nu, m} a_{\nu, m}\left(2^{k} x\right), \quad x \in \mathbb{R}^{n},
$$

would represent an atomic decomposition of $f\left(2^{k} x\right)$. This can be seen by setting

$$
g_{k}(x)=\sum_{\nu, m} \lambda_{\nu, m} 2^{k\left(\sigma_{p}-\frac{n}{p}\right)} 2^{-k\left(\sigma_{p}-\frac{n}{p}\right)} a_{\nu, m}\left(2^{k} x\right)=\sum_{\nu, m} \lambda_{\nu, m}^{k} a_{\nu, m}^{k}(x),
$$

where $a_{\nu, m}^{k}(x)=2^{-k\left(\sigma_{p}-\frac{n}{p}\right)} a_{\nu, m}\left(2^{k} x\right) \sim \tilde{a}_{\nu+k, m}(x)$, since

$$
\begin{gathered}
\operatorname{supp} a_{\nu, m}^{k} \subset Q_{\nu+k, m}, \\
\left|\mathrm{D}^{\alpha} a_{\nu, m}^{k}(x)\right|=2^{-k\left(\sigma_{p}-\frac{n}{p}\right)+k|\alpha|}\left|\mathrm{D}^{\alpha} a_{\nu, m}(x)\right| \leq 2^{-(\nu+k)\left(\sigma_{p}-\frac{n}{p}\right)+(\nu+k)|\alpha|} .
\end{gathered}
$$

Therefore we obtain

$$
\left\|g_{k}\left|B_{p, q}^{\sigma_{p}}\|\leq\| \lambda^{k}\right| b_{p, q}\right\|=2^{k\left(\sigma_{p}-\frac{n}{p}\right)}\left\|\lambda\left|b_{p, q}\left\|=2^{-n k}\right\| \lambda\right| b_{p, q}\right\|,
$$

yielding

$$
\left\|f\left(2^{k} \cdot\right)\left|B_{p, q}^{\sigma_{p}}\left\|\leq c 2^{-n k}\right\| f\right| B_{p, q}^{\sigma_{p}}\right\| .
$$

But we know by Theorem 2.1 that this is not true in general if $q<\infty$.

Remark 3.11. Using a different argument it is possible to show that for $q=\infty$ the first moment conditions are indispensable as well.

Let $a \in S\left(\mathbb{R}^{n}\right)$ with $\operatorname{supp} a \subset\{y:|y|<1\}$ and $\int_{\mathbb{R}^{n}} a(y) \mathrm{d} y=1$. Then

$$
a_{j}(x)=2^{j n} a\left(2^{j} x\right), \quad j \in \mathbb{N}_{0}, \quad x \in \mathbb{R}^{n},
$$


satisfy the support and growth assumptions for normalized atoms in

$$
B_{p, q}^{s}\left(\mathbb{R}^{n}\right) \quad \text { with } \quad 0<p \leq \infty, \quad 0<q \leq \infty, \quad s=n\left(\frac{1}{p}-1\right) .
$$

But

$$
f^{J}(x)=\sum_{j=0}^{J} a_{j}(x), \quad J \in \mathbb{N},
$$

does not converge in $S^{\prime}\left(\mathbb{R}^{n}\right)$. This follows from

$$
\left(f^{J}, \psi\right)=\sum_{j=0}^{J} \int_{\mathbb{R}^{n}} a_{j}(x) \psi(x) \mathrm{d} x=J+1,
$$

for any $\psi \in S\left(\mathbb{R}^{n}\right)$ with $\psi(x)=1$ if $|x| \leq 1$. Hence (27) cannot be an atomic representation for the spaces in (26) with $q=\infty$ even though

$$
\left\|\lambda \mid b_{p, \infty}\right\|=\sup _{j=0, \ldots, J} \lambda_{j}=1 .
$$

Of interest for our considerations is only the case $0<p<1$. Then (27) converges in $L_{p}\left(\mathbb{R}^{n}\right)$, since, for $J^{\prime}>J$,

$$
\begin{aligned}
\left\|f^{J^{\prime}}-f^{J} \mid L_{p}\left(\mathbb{R}^{n}\right)\right\|^{p} & \leq \sum_{j=J+1}^{J^{\prime}} \int_{\mathbb{R}^{n}}\left|a_{j}(x)\right|^{p} \mathrm{~d} x \\
& =\left\|a \mid L_{p}\left(\mathbb{R}^{n}\right)\right\|^{p} \sum_{j=J+1}^{J^{\prime}} 2^{j n p-j n} \sim 2^{-J n(1-p)} .
\end{aligned}
$$

Furthermore,

$$
\lim _{J \rightarrow \infty} f^{J}(x) \sim|x|^{-n} \quad \text { near the origin. }
$$

Acknowledgements. This gives me the opportunity to thank J. Vybíral for many valuable discussions and comments on the subject as well as D. D. Haroske and H. Triebel for their support.

\section{References}

[1] C. Bennett and R. Sharpley, Interpolation of operators, Pure and Applied Mathematics, vol. 129, Academic Press Inc., Boston, MA, 1988.

[2] H.-Q. Bui, M. Paluszyński, and M. H. Taibleson, A maximal function characterization of weighted Besov-Lipschitz and Triebel-Lizorkin spaces, Studia Math. 119 (1996), no. 3, 219246 . 
[3] Characterization of the Besov-Lipschitz and Triebel-Lizorkin spaces: The case $q<1$, J. Fourier Anal. Appl. 3 (1997), 837-846. Proceedings of the conference dedicated to Professor Miguel de Guzmán (El Escorial, 1996).

[4] R. A. DeVore and G. G. Lorentz, Constructive approximation, Grundlehren der Mathematischen Wissenschaften, vol. 303, Springer-Verlag, Berlin, 1993.

[5] D. E. Edmunds and H. Triebel, Function spaces, entropy numbers, differential operators, Cambridge Tracts in Mathematics, vol. 120, Cambridge University Press, Cambridge, 1996.

[6] D. D. Haroske and C. Schneider, Besov spaces with positive smoothness on $\mathbb{R}^{n}$, embeddings and growth envelopes, J. Approx. Theory, to appear.

[7] L. I. Hedberg and Yu. V. Netrusov, An axiomatic approach to function spaces, spectral synthesis, and Luzin approximation, Mem. Amer. Math. Soc. 188 (2007), no. 882, vi+97.

[8] Yu. V. Netrusov, Sets of singularities of functions in spaces of Besov and Lizorkin-Triebel type, Trudy Mat. Inst. Steklov. 187 (1989), 162-177 (Russian); English transl., Proc. Steklov Inst. Math. 187 (1990), no. 3, 185-203. Theory and Applications of Differential Functions of Several Variables XIII.

[9] V. S. Rychkov, On a theorem of Bui, Paluszyński, and Taibleson, Tr. Mat. Inst. Steklova 227 (1999), 286-298 (Russian); English transl., Proc. Steklov Inst. Math. 4 (227) (1999), 280-292.

[10] W. Sickel and H. Triebel, Hölder inequalities and sharp embeddings in function spaces of $B_{p q}^{s}$ and $F_{p q}^{s}$ type, Z. Anal. Anwendungen 14 (1995), no. 1, 105-140.

[11] 亡̀. A. Storoženko and P. Osval'd, Jackson's theorem in the spaces $L^{p}\left(\mathbf{R}^{k}\right), 0<p<1$, Sibirsk. Mat. Ž. 19 (1978), no. 4, 888-901.

[12] H. Triebel, Interpolation theory, function spaces, differential operators, North-Holland Mathematical Library, vol. 18, North-Holland Publishing Co., Amsterdam, 1978.

[13] _ Theory of function spaces, Monographs in Mathematics, vol. 78, Birkhäuser Verlag, Basel, 1983.

[14] _ Theory of function spaces, II, Monographs in Mathematics, vol. 84, Birkhäuser Verlag, Basel, 1992.

[15] _ Fractals and spectra, Monographs in Mathematics, vol. 91, Birkhäuser-Verlag, Basel, 1997.

[16] _ The structure of functions, Monographs in Mathematics, vol. 97, Birkhäuser Verlag, Basel, 2001.

[17] _ Theory of function spaces, III, Monographs in Mathematics, vol. 100, Birkhäuser Verlag, Basel, 2006.

[18] J. Vybíral, Dilation operators and samping numbers, J. Funct. Spaces Appl., to appear. 